\title{
Application of neutrophil to lymphocyte ratio to identify CT-negative cerebral infarction with nonfocal symptoms
}

\author{
Ming-Liang Wang, Meng-Meng Yu, Wen-Bin Li, Yue-Hua Li \\ Department of Radiology, Shanghai Jiao Tong University Affiliated Sixth People's Hospital, Shanghai Jiao Tong University School of Medicine, \\ Shanghai, China \\ Contributions: (I) Conception and design: ML Wang, YH Li; (II) Administrative support: YH Li; (III) Provision of study materials or patients: None; \\ (IV) Collection and assembly of data: ML Wang, MM Yu, WB Li; (V) Data analysis and interpretation: ML Wang, MM Yu, YH Li; (VI) Manuscript \\ writing: All authors; (VII) Final approval of manuscript: All authors. \\ Correspondence to: Yue-Hua Li. No. 600, Yi Shan Road, Shanghai 200233, China. Email: liyuehua312@163.com.
}

\begin{abstract}
Background: The neutrophil to lymphocyte ratio (NLR) has emerged as a strong predictor of mortality in stroke patients. Our study aimed to investigate the correlation between NLR and cerebral infarction with nonfocal symptoms confirmed by diffusion-weighted imaging (DWI) (+).

Methods: A total of 439 patients with nonfocal stroke symptoms with CT-negative findings were included from January 1 to December 31, 2018. All patients underwent a head MRI examination within seven days following a head CT examination. The patients' demographics, medical history, presenting symptoms, and stroke location were recorded. Logistic regression and receiver operating characteristic (ROC) curve analysis were used to identify variables with a significant association with cerebral infarction.

Results: Cerebral infarction was detected in 79 (18\%) patients confirmed by DWI(+), located mostly in the cerebellum (40.51\%). Dizziness $(85.19 \%)$ was the most common symptom. The cerebral infarction group showed a higher prevalence of hypertension $(\mathrm{P}<0.0001)$, diabetes mellitus $(\mathrm{P}<0.0001)$, and smoking status $(\mathrm{P}=0.001)$ than non-cerebral infarction group. The NLR $(\mathrm{P}<0.0001)$ was higher in the cerebral infarction group. There was no significant difference in NIHSS $(\mathrm{P}=0.09)$. Logistic analysis revealed that male gender $(\mathrm{P}=0.046)$, a history of hypertension $(\mathrm{P}=0.001)$, diabetes mellitus $(\mathrm{P}=0.001)$, smoking $(\mathrm{P}=0.023)$, and NLR $(\mathrm{P}<0.0001)$ were the best predictors of cerebral infarction. When integrating sex, hypertension, diabetes mellitus, smoking and NLR, the area under ROC value of the combined method was 0.785 , higher than any separate parameters $(\mathrm{P}<0.05)$.

Conclusions: NLR combined with male gender, a history of hypertension, diabetes mellitus, and smoking, could predict DWI-confirmed cerebral infarction with nonfocal neurologic symptoms with high diagnostic accuracy.
\end{abstract}

Keywords: Neutrophil to lymphocyte ratio (NLR); diffusion-weighted imaging (DWI); cerebral infarction; nonfocal neurologic symptom

Submitted Feb 16, 2020. Accepted for publication Sep 14, 2020.

doi: $10.21037 /$ atm-20-1640

View this article at: http://dx.doi.org/10.21037/atm-20-1640

\section{Introduction}

Missed diagnosis of cerebral infarction has been frequently reported ranging from $2-26 \%$ especially found in posterior circulation stroke with nonfocal symptoms such as altered mental status, dizziness, and headache (1-3). The failure to recognize an acute cerebral infarction could delay the timely secondary prevention treatment and may bring a disastrous outcome to the patients. The NIHSS scale (4) and FAST assessments (5) were reported to be less sensitive in diagnosing posterior circulation stroke. Head computed tomography (CT) is also insensitive to detect brain stem lesions, especially in acute cerebral infarction. 


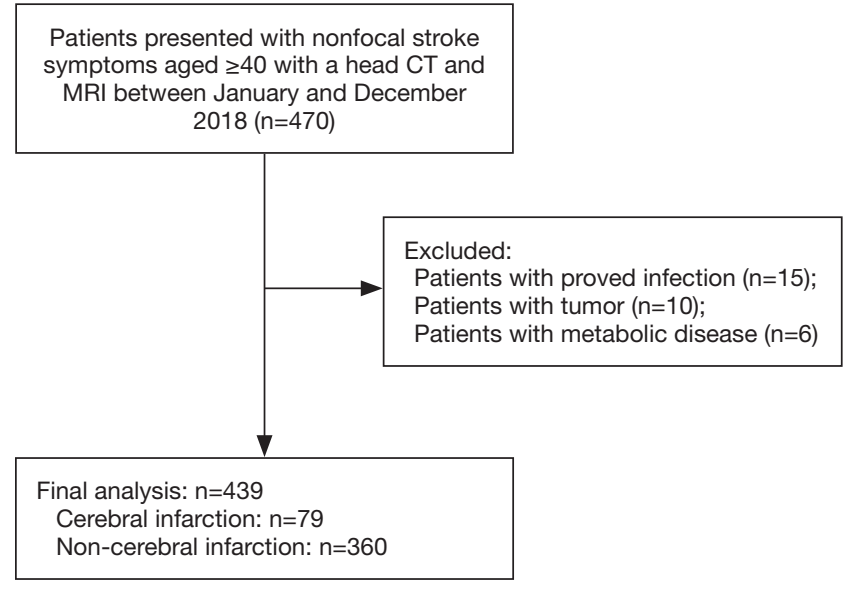

Figure 1 Flow chart of patient inclusion and exclusion criteria.

Diffusion-weighted imaging (DWI) sequence in MRI is proven to be useful in the detection of cerebral infarction $(6,7)$, even in unselected suspected patients (8). However, MRI scanning of all patients with suspected cerebral infarction was not cost-effective $(9,10)$. Furthermore, it may not always be possible to perform a scan due to unavailability. The newly published Guidelines for the Early Management of Patients with Acute Cerebral Infarction also indicated this problem (11). Therefore, finding a reliable biomarker to identify the most probable cerebral infarction patients to undergo head MRI is needed in order to not only reduce the misdiagnosis of cerebral infarction but also reduce unnecessary usage of limited medical resources.

The neutrophil to lymphocyte ratio (NLR), which is calculated as the ratio of neutrophil to leukocyte, has been used as a marker of systemic inflammation in clinical practice. Previous studies have proved that the pathophysiology of cerebral infarction involved an inflammatory process not only in local ischemic brain but also in the peripheral inflammatory system $(12,13)$. Neutrophils have been reported to increase within 15 minutes of the brain ischemia and could surround the damaged cerebral vessels (14). At present, NLR has been reported to be a predictor of short-term mortality in acute stroke patients (15) and is even associated with increased risk of new cerebral infarction and composite events in minor stroke or transient ischemic attack $(16,17)$. However, no study has investigated the value of NLR in the identification of cerebral infarction with nonfocal symptoms.

As the routine complete blood count test is an easy and convenient test to perform, we wondered whether NLR could be used as a biomarker to help in the identification of cerebral infarction with nonfocal stroke symptoms for further head MRI assessment. Our primary aim was to analyze the characteristics of CT-negative cerebral infarction with nonfocal stroke symptoms, and the secondary aim was to investigate whether NLR is associated with CT-negative cerebral infarction with nonfocal stroke symptoms.

We present the following article in accordance with the STROBE reporting checklist (available at http://dx.doi. org/10.21037/atm-20-1640).

\section{Methods}

\section{Study population}

The study was conducted in accordance with the Declaration of Helsinki (as revised in 2013). This study was approved by the institutional review board of Shanghai Sixth People's Hospital (No. YS-2019-001). As this study was retrospective, the requirement for informed consent was waived. From January 1, 2018, to December 31, 2018 , adult patients $\geq 40$ years old with nonfocal stroke symptoms presenting to the Emergency Department (ED) and having a head CT were included (Figure 1). Nonfocal stroke symptoms were defined as common stroke confusing symptoms including altered consciousness, dizziness, headache, and memory loss, as reported in previous studies (18-20). In our hospital, patients with nonfocal stroke symptoms were firstly evaluated by an ED neurologist. NIHSS scale was done to assess the severity. A head CT examination was ordered for the suspected stroke detection or other unexplained neurologic symptoms. A further head MRI may be ordered for detecting cerebral infarction or excluding other severe brain diseases while the patient agreed to have a head MRI and had no MRI contraindications. The final diagnosis of cerebral infarction was diagnosed by neurologists based on the detailed medical history, neurological examination, clinical assessment (NIHSS score), and imaging results including brain CT and MR-DWI results.

The following inclusion criteria were applied: (I) adult patients who were more than 40 years old and had nonfocal stroke symptoms; (II) patients should have a head CT examination and a routine complete blood count test within 24 hours, followed by a head MRI examination within seven days. We excluded the following subjects: patients with proven infection and inflammatory disease on admission; those with evidence of brain hemorrhage, tumors, and 
traumatic disease observed on CT imaging; patients with a history of cancer; patients with severe hepatic and renal diseases; patients with acute metabolic diseases; and patients with psychiatric disorders.

\section{Brain MRI protocols}

MRI was performed by trained technicians on $3.0 \mathrm{~T}$ MRI scanners (Magnetom Verio ${ }^{\mathrm{TM}}$; Siemens Medical Systems, Erlangen, Germany; Magnetom Skra ${ }^{\mathrm{TM}}$; Siemens Medical Systems, Erlangen, Germany; or Ingenia ${ }^{\mathrm{TM}}$; Philips, Best, The Netherlands). The brain MRI protocol included axial T1-weighted, axial T2-weighted, axial fluid-attenuated inversion recovery, axial diffusion-weighted, and sagittal T1-weighted sequences.

\section{Data collection}

Demographics and clinical information including age, sex, and symptoms of each patient were collected. Medications and medical history of hypertension, diabetes, coronary heart disease, atrial fibrillation, smoking, and alcoholism were also collected from the medical record. Complete blood count analysis, including total white blood cell (WBC), neutrophil, and lymphocyte was conducted using an auto-analyzer in our hospital. The NLR value was calculated as the ratio of absolute neutrophil to lymphocyte.

Negative head CT result was defined as having no evidence of intracranial hemorrhage, recent obvious ischemia, or mass lesion. Two experienced neuroradiologists (with 12- and 15-year experience in neuroimaging) who were blinded to all clinical information and the MR images interpreted the head CT image. Any disagreements were resolved by consensus. MRI was interpreted by another experienced neuroradiologist (with 8-year experience in neuroimaging), mainly focused on any brain area showing high signal on DWI sequence.

\section{Statistical analysis}

All statistical analysis in this study was performed using SPSS for Microsoft Windows software (version 20.0, IBM). The Kolmogorov-Smirnov test was used to test normality for continuous variables. The Mann-Whitney $U$ test and chi-square test were used to analyze the difference of demographic and clinical characteristics between the cerebral infarction group and non-cerebral infarction group.
Logistic regression was used to assess the relationship between DWI-confirmed cerebral infarction and the study variables by the backward stepwise procedure. The logistic regression model included variables with $\mathrm{P}<0.05$ in the univariate analysis. Variables that were significant in the logistic analysis were included for further receiver operating characteristic (ROC) curve analysis. The combined performance of these variables was investigated using binary logistic regression (details are provided in Supplementary). The optimal cutoff values for various parameters were determined using the maximum sum of sensitivity and specificity in the ROC curve analysis. The area under ROC (AUC) was compared using the DeLong method (21). $\mathrm{P}<0.05$ was considered statistically significant.

\section{Results}

\section{Clinical characteristics of the study participants}

The clinical characteristics of the study population are presented in Table 1. A total of 439 patients in the ED with nonfocal stroke symptoms with CT-negative findings were included in this study (Figure 1). Median (interquartile range) age of the study patients was 69 years (63-78 years), and 231 patients $(52.62 \%)$ were women. The most common symptom was dizziness $(85.19 \%)$, followed by altered consciousness $(7.06 \%)$, headache $(5.47 \%)$, and memory impairment (2.28\%). As for medical history, hypertension $(59.68 \%)$ was most frequently observed, followed by diabetes mellitus (23.69\%), coronary heart disease (9.79\%), and atrial fibrillation (2.96\%). Furthermore, $12.76 \%$ and $10.02 \%$ of patients had a history of smoking and alcoholism, respectively.

Seventy-nine (18\%) patients had a confirmed diagnosis of cerebral infarction based on clinical characteristics and DWI(+) findings. The most common location of cerebral infarction was the cerebellum (40.51\%), followed by the anterior circulation area (32.91\%), brain stem (26.58\%), and thalamus (7.59\%) (Figure 2). Notably, nine patients had cerebral infarction in two different locations, and one patient had cerebral infarction in three different locations. Among the non-cerebral infarction patients, the symptoms were diagnosed as secondary symptoms resulting from hypertension (188, 52.22\%), diabetes (36,10\%), peripheral vertigo $(21,5.83 \%)$ vertebrobasilar insufficiency ( 11 , $3.06 \%$ ), mild cognitive impairment (6,1.67\%), and no specific diagnosis $(27.22 \%)$. 
Table 1 Demographics of the study participants

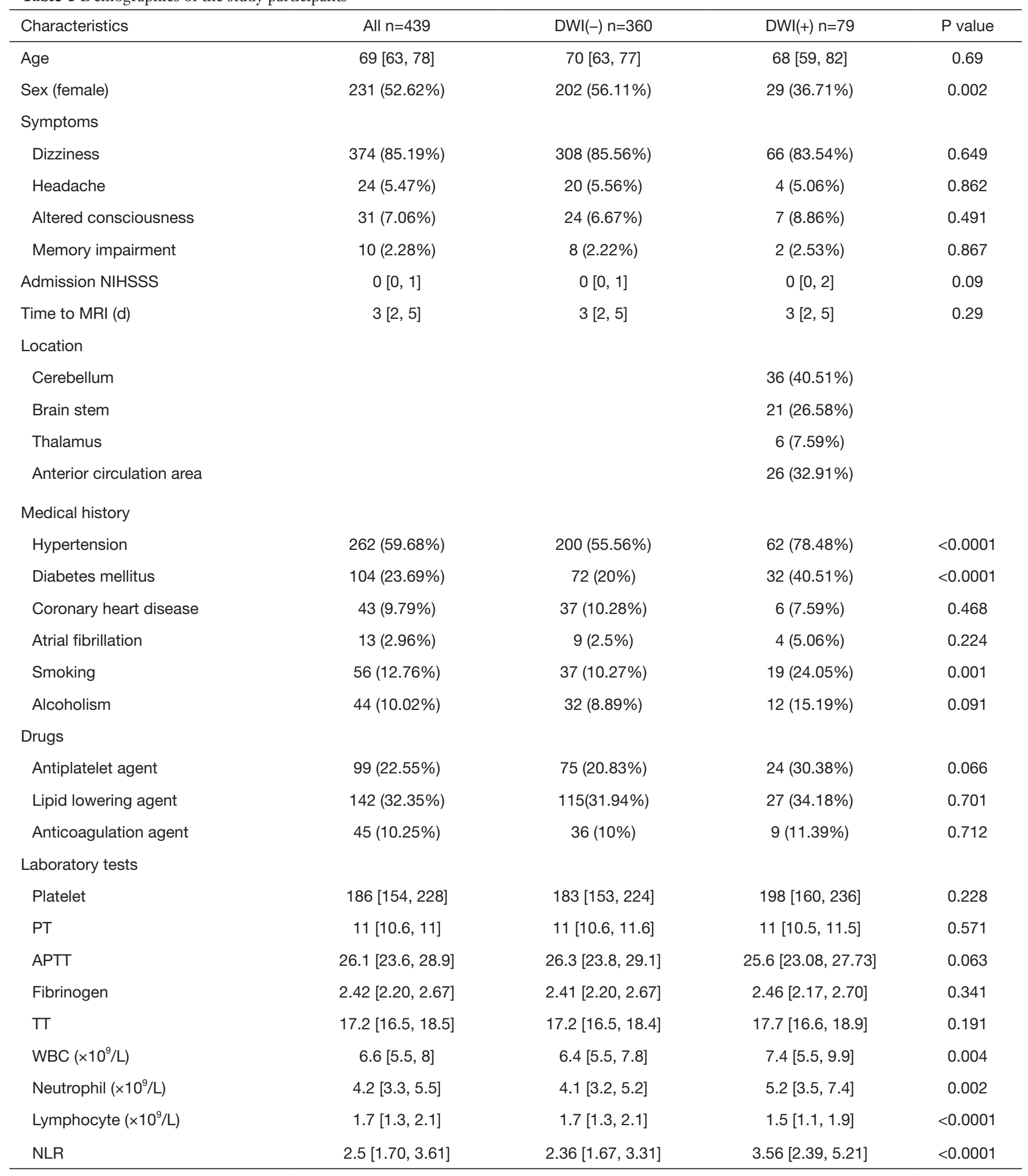

Data are presented as $\mathrm{n}(\%)$ or median [interquartile range]. NIHSS, National Institutes of Health Stroke Scale; MRI, magnetic resonance imaging; WBC, white blood cell; NLR, neutrophil-to-lymphocyte ratio; PT, prothrombin time; APTT, activated partial thromboplastin time; TT, thrombin time. 

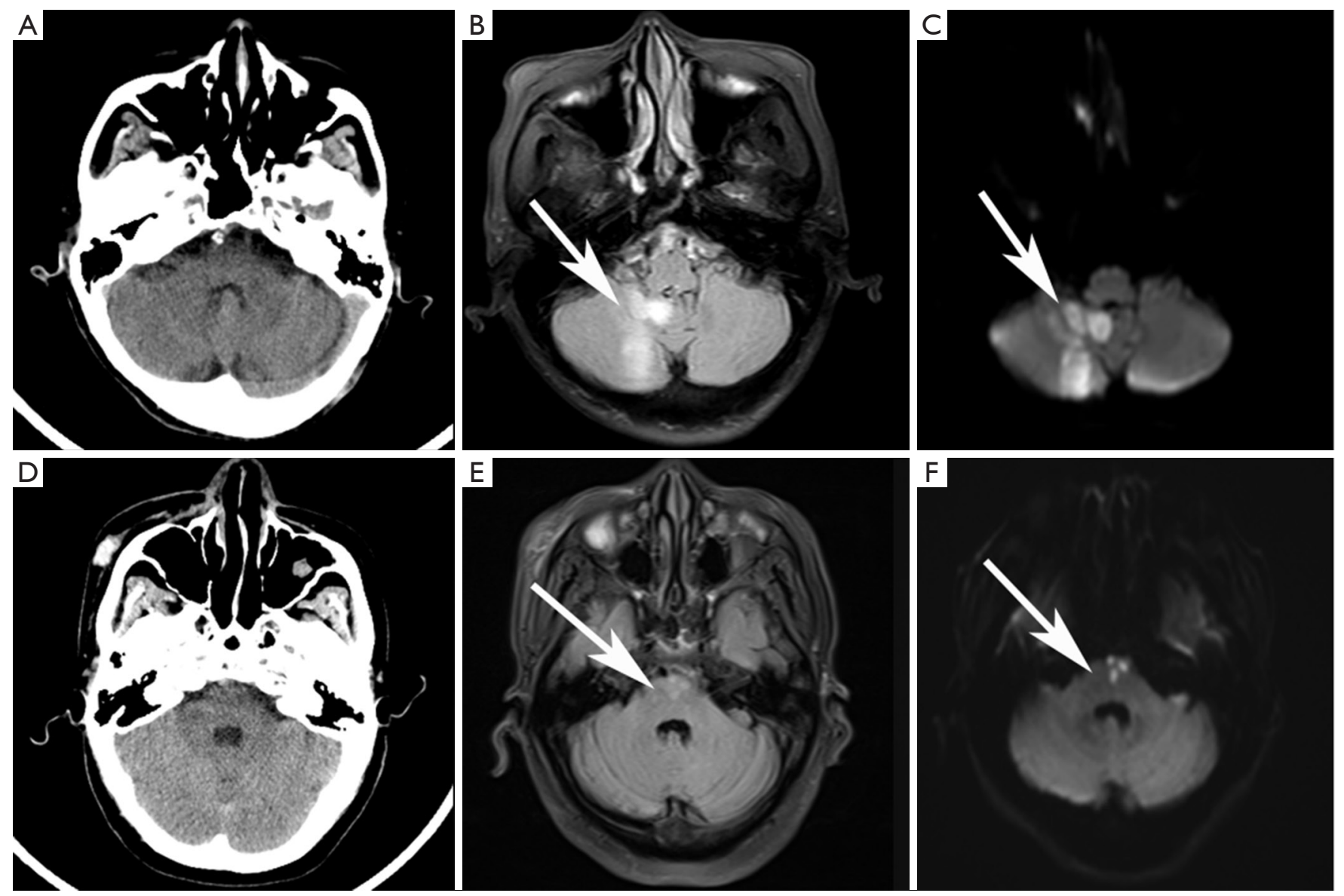

Figure 2 Typical images of cerebral infarction with nonfocal symptoms confirmed by diffusion imaging (DWI)(+). The first patient was a 64 male patient with symptoms of dizziness and had hypertension and diabetes. The NLR value was 8.4. The CT result was negative (A), with brain lesion in the right cerebellum in Flair sequence (B) and DWI sequence (C). The second patient was a 68-male patient with symptoms of dizziness and had hypertension. The NLR value was 5.25. The CT result was negative (D), with small brain lesions in the pons in Flair sequence (E) and DWI sequence (F). The arrows indicate the location of cerebral infarction.

\section{Correlation between NLR and cerebral infarction with nonfocal stroke symptoms}

Table 1 also shows the comparison of clinical characteristics between the cerebral infarction group and the noncerebral infarction group. More male than female patients had cerebral infarction $(\mathrm{P}=0.002)$. The cerebral infarction group showed a higher prevalence of hypertension $(\mathrm{P}<0.0001)$, diabetes mellitus $(\mathrm{P}<0.0001)$, and smoking status $(\mathrm{P}=0.001)$ than the non-cerebral infarction group. The NLR $(\mathrm{P}<0.0001)$ and $\mathrm{WBC}(\mathrm{P}=0.004)$ and neutrophil $(\mathrm{P}=0.002)$ counts were higher, while the lymphocyte $(\mathrm{P}<0.0001)$ count was lower in the cerebral infarction group than the non-cerebral infarction group. Furthermore, there was no significant difference in

\section{NIHSS score $(\mathrm{P}=0.09)$.}

Table 2 shows the results of the logistic regression analysis with all clinical characteristics and NLR. Our study revealed that male gender (odds ratio, 1.767; $\mathrm{P}=0.046$ ); a history of hypertension (odds ratio, 2.888; $\mathrm{P}=0.001$ ), diabetes mellitus (odds ratio, 2.679; $\mathrm{P}=0.001$ ), smoking (odds ratio, 2.257; $\mathrm{P}=0.023$ ); and NLR (odds ratio, $1.420 ; \mathrm{P}<0.0001$ ) were the best predictors for cerebral infarction.

\section{Diagnostic performance of NLR for identifying cerebral infarction with nonfocal stroke symptoms}

Table 3 shows the diagnostic performance of NLR and other clinical parameters separately and synthetically. At 
Table 2 Logistic regression analysis for cerebral infarction confirmed by diffusion-weighted imaging (+)

\begin{tabular}{lccc}
\hline Variables & Odds ratio & $95 \% \mathrm{Cl}$ & $\mathrm{P}$ value \\
\hline Sex (male) & 1.767 & $1.010-3.091$ & 0.046 \\
Hypertension & 2.888 & $1.514-5.507$ & 0.001 \\
Diabetes mellitus & 2.679 & $1.511-4.751$ & 0.001 \\
Smoking & 2.257 & $1.117-4.561$ & 0.023 \\
NLR & 1.420 & $1.248-1.615$ & $<0.0001$ \\
\hline
\end{tabular}

NLR, neutrophil-to-lymphocyte ratio.

Table 3 Diagnostic performance of NLR for predicting cerebral infarction with nonfocal symptom when using best cutoff values

\begin{tabular}{lccccc}
\hline Variables & AUC (95\% Cl) & Best cutoffs & TP/FP/TN/FN & $\begin{array}{c}\text { Sensitivity, } \\
\%(95 \% \text { Cl })\end{array}$ \\
\hline Sex (male) & $0.597(0.549,0.643)$ & 1 & $50 / 158 / 202 / 29$ & $63.29(51.7,73.9)$ & $\begin{array}{c}\text { Specificity, } \\
\%(95 \% \mathrm{Cl})\end{array}$ \\
Hypertension (HT) & $0.615(0.567,0.660)$ & 1 & $62 / 200 / 160 / 17$ & $78.48(67.8,86.9)$ & $44.44(39.2,49.7)$ \\
Diabetes mellitus (DM) & $0.603(0.555,0.649)$ & 1 & $32 / 72 / 288 / 47$ & $40.51(29.6,52.1)$ & $80.0(75.5,84.0)$ \\
Smoking (SM) & $0.569(0.521,0.616)$ & 1 & $19 / 37 / 323 / 60$ & $24.05(15.1,35.0)$ & $89.72(86.1,92.7)$ \\
NLR & $0.691(0.646,0.734)$ & $>2.3529$ & $62 / 180 / 180 / 17$ & $78.48(67.8,86.9)$ & $50.0(44.7,55.3)$ \\
NLR + male + HT + DM + SM & $0.785(0.744,0.823)$ & $>0.15173$ & $61 / 120 / 240 / 18$ & $77.22(66.4,85.9)$ & $66.67(61.5,71.5)$ \\
\hline
\end{tabular}

NLR, neutrophil-to-lymphocyte ratio; FN, false negative; FP, false positive; TN, true negative; TP, true positive.

the cutoff value of 2.35 , NLR achieved a sensitivity value of $78.48 \%$ and a specificity value of $50.00 \%$ in diagnosing cerebral infarction. Figure 3 depicts the comparison of AUC of the study variables and the combined effect. NLR had an AUC value of 0.691 for identifying cerebral infarction, higher than sex $(\mathrm{P}=0.0341)$, and smoking $(\mathrm{P}=0.002)$. When integrating sex, hypertension, diabetes mellitus, smoking, and NLR, the AUC value of the combined method was 0.785 , higher than any separate parameters $(\mathrm{P}<0.05)$.

\section{Discussion}

Accurate diagnosis of cerebral infarction, especially in suspected cases with nonfocal symptoms, is of paramount importance to ensure timely and appropriate treatment. Our study found that the most common nonfocal stroke symptom was dizziness, and the most common location of cerebral infarction was the cerebellum. NLR combined with male gender and a history of hypertension, diabetes mellitus, and smoking were associated with CT-negative cerebral infarction with nonfocal neurologic symptoms.

In our study, the most common nonfocal symptom in suspected cerebral infarction was dizziness, followed by altered consciousness, headache, and memory impairment. Dizziness is a common chief complaint in the ED, responsible for about 4.4 million ED visits in the United States annually (22). Although strokes only account for roughly $3-5 \%$ of all ED patients presenting with dizziness (23), the differential diagnosis is a significant and challenging task. HINTS test is a useful tool to discriminate central from peripheral dizziness or vertigo (24). However, there are also limitations to the use of HINTS. HINTS could not be used in acute transient vestibular syndrome patients, whose vestibular symptoms or signs had already resolved when the patients arrived at the hospital (25). Furthermore, the HINTS test maybe poorly performed in the ED department with a non-neuro-ophthalmologist. Altered consciousness, headache, and memory impairment were less commonly observed, but the differential diagnosis was tricky as all these symptoms were frequently unaccompanied by more obvious neurological signs of cerebral infarction (26-28).

The MR-DWI has been proven to be sensitive in the detection of cerebral infarction (6,7). In our study, $18 \%$ 


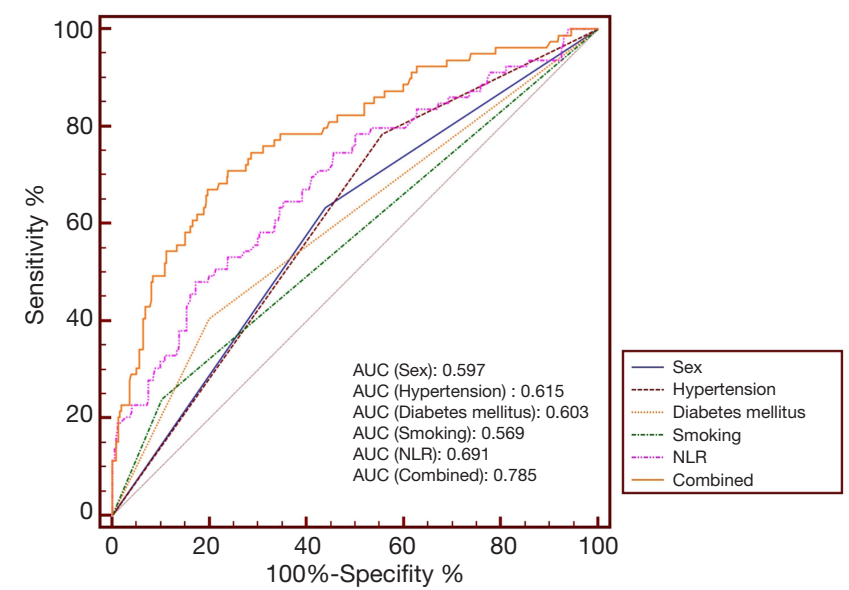

Figure 3 ROC curve analysis of sex, hypertension, diabetes mellitus, smoking, and NLR for identifying cerebral infarction. NLR had an AUC value of 0.691 for identifying cerebral infarction, higher than sex $(\mathrm{P}=0.0341)$, and smoking $(\mathrm{P}=0.002)$. The AUC value of the combined method was 0.785 , higher than any separate parameters $(\mathrm{P}<0.05)$.

of patients with nonfocal neurologic symptoms were diagnosed as cerebral infarction confirmed by DWI(+). A previous study also found that $14 \%$ of low-risk population patients without motor/speech neurological symptoms had MRI DWI(+) results (29). In our study, the most common location of the cerebral infarction was the cerebellum. This was similar to previous studies, which indicated that nonfocal symptoms including dizziness and headache were more frequent in patients with posterior circulation stroke $(24,26,27,30,31)$. However, our study also found that $32.91 \%$ of patients had anterior circulation cerebral infarction. Analyzing the characteristics of these patients, we found most of the anterior circulation cerebral infarction in our study was minor type and most of the patients had a symptom of altered consciousness, which was difficult to obtain an accurate history or perform a detailed neurologic examination. Therefore, patients with altered consciousness should be paid special attention.

We further investigated whether NLR, an inflammatory indicator, could help to predict cerebral infarction confirmed by DWI(+). Our results showed that the cerebral infarction group had higher NLR than the non-cerebral infarction group. A previous study revealed that early elevated NLR was associated with remote DWI(+) lesions in acute intracerebral hemorrhage (32). It is well established that inflammation is acutely involved in the process of ischemic stroke (33). Neutrophils have been reported to increase within 15 minutes of the brain ischemia and could surround the damaged cerebral vessels; they infiltrate after 6-8 hours, peaking at 24-48 hours, while lymphocytes decrease after an ischemic stroke, peaking at 24-48 hour (14). Elevated NLR may represent a marker of tissue inflammation severity due to ischemic injury (34), while the injury was too early to be seen in the CT. A previous study even found that elevated NLR was associated with cerebral infarction volume in DWI (12). Thus, it was conceivable that NLR could elevate in CT-negative cerebral infarction.

Apart from NLR, we also analyzed the value of clinical characteristics in predicting cerebral infarction. Our study found that male sex, hypertension, diabetes mellitus, and smoking status were more commonly observed in the cerebral infarction group. This was reasonable as these medical histories are known risk factors for ischemic stroke even in patients with nonfocal symptoms (35-37). A previous study also found that the presence of hypertension and diabetes mellitus could be used to lower the threshold for ordering a head MRI examination for suspected ischemic stroke with nonfocal symptoms (18).

Further logistic regression analysis revealed that male sex, a history of hypertension, diabetes mellitus, and smoking, and NLR were the best predictors for cerebral infarction. When integrating sex, hypertension, diabetes mellitus, smoking, and NLR, the AUC value of the combined method was 0.785 , higher than any separate parameters. As the routine complete blood count is an easy and convenient test to perform, combined with the above-mentioned factors, NLR could be used to help select the most probable suspected cerebral infarction case to have a head MRI for further assessment and confirmation.

Our study had some limitations. First, the study sample may have a selection bias. In our study, head CT and MRI were ordered for the evaluation of suspected cerebral infarction. However, the criteria for ordering head CT and MRI may be different for different hospitals and ED neurologists. Second, the MRI scanning interval time was a little heterogeneous within seven days after head CT. The treatment and condition from the time of patient admission to the MRI examination may affect the NLR value and DWI positivity. However, there was no significant difference in the MRI scanning interval time between the cerebral infarction group and the non-cerebral infarction group. Third, the sample size in this study was small. Prospective studies with larger samples are needed to 
validate our findings.

In conclusion, the most common nonfocal stroke symptom was dizziness, and the most common location of the cerebral infarction was the cerebellum. NLR combined with the male gender, a history of hypertension, diabetes mellitus, and smoking, could predict DWI-confirmed cerebral infarction with nonfocal neurologic symptoms.

\section{Acknowledgments}

Funding: This study was supported by National Natural Science Foundation of China $(81901727,81871329$, 81671673), New developing and Frontier Technologies of Shanghai Shen Kang Hospital Development Center (SHDC12018117), Shanghai Municipal Education Commission-Gaofeng Clinical Medicine Grant Support (2016427), Excellent discipline leader of Shanghai Municipal Planning Commission (2017BR041), and Shanghai key discipline of medical imaging (2017ZZ02005).

\section{Footnote}

Reporting Checklist: The authors have completed the STROBE reporting checklist. Available at http://dx.doi. org/10.21037/atm-20-1640

Data Sharing Statement: Available at http://dx.doi. org/10.21037/atm-20-1640

Peer Review File: Available at http://dx.doi.org/10.21037/ atm-20-1640

Conflicts of Interest: All authors have completed the ICMJE uniform disclosure form (available at http://dx.doi. org/10.21037/atm-20-1640). The authors have no conflicts of interest to declare.

Ethical Statement: The authors are accountable for all aspects of the work in ensuring that questions related to the accuracy or integrity of any part of the work are appropriately investigated and resolved. The study was conducted in accordance with the Declaration of Helsinki (as revised in 2013). The study was approved by the Ethics Committee of Shanghai Sixth People's Hospital (No. YS2019-001). As this study was retrospective, the requirement for informed consent was waived.
Open Access Statement: This is an Open Access article distributed in accordance with the Creative Commons Attribution-NonCommercial-NoDerivs 4.0 International License (CC BY-NC-ND 4.0), which permits the noncommercial replication and distribution of the article with the strict proviso that no changes or edits are made and the original work is properly cited (including links to both the formal publication through the relevant DOI and the license). See: https://creativecommons.org/licenses/by-nc-nd/4.0/.

\section{References}

1. Arch AE, Weisman DC, Coca S, et al. Missed Ischemic Stroke Diagnosis in the Emergency Department by Emergency Medicine and Neurology Services. Stroke 2016;47:668-73.

2. Richoz B, Hugli O, Dami F, et al. Acute stroke chameleons in a university hospital: Risk factors, circumstances, and outcomes. Neurology 2015;85:505-11.

3. Liberman AL, Prabhakaran S. Stroke Chameleons and Stroke Mimics in the Emergency Department. Curr Neurol Neurosci Rep 2017;17:15.

4. Heldner MR, Zubler C, Mattle HP, et al. National Institutes of Health stroke scale score and vessel occlusion in 2152 patients with acute ischemic stroke. Stroke 2013;44:1153-7.

5. Wolters FJ, Li L, Gutnikov SA, et al. Medical Attention Seeking After Transient Ischemic Attack and Minor Stroke Before and After the UK Face, Arm, Speech, Time (FAST) Public Education Campaign: Results From the Oxford Vascular Study. JAMA Neurol 2018;75:1225-33.

6. Mullins ME, Schaefer PW, Sorensen AG, et al. CT and conventional and diffusion-weighted MR imaging in acute stroke: study in 691 patients at presentation to the emergency department. Radiology 2002;224:353-60.

7. Chalela JA, Kidwell CS, Nentwich LM, et al. Magnetic resonance imaging and computed tomography in emergency assessment of patients with suspected acute stroke: a prospective comparison. Lancet 2007;369:293-8.

8. Brunser AM, Hoppe A, Illanes S, et al. Accuracy of diffusion-weighted imaging in the diagnosis of stroke in patients with suspected cerebral infarct. Stroke 2013;44:1169-71.

9. Brazzelli M, Sandercock PA, Chappell FM, et al. Magnetic resonance imaging versus computed tomography for detection of acute vascular lesions in patients presenting 
with stroke symptoms. Cochrane Database Syst Rev 2009;(4):CD007424.

10. Wardlaw J, Brazzelli M, Miranda H, et al. An assessment of the cost-effectiveness of magnetic resonance, including diffusion-weighted imaging, in patients with transient ischaemic attack and minor stroke: a systematic review, meta-analysis and economic evaluation. Health Technol Assess 2014;18:1-368, v-vi.

11. Powers WJ, Rabinstein AA, Ackerson T, et al. 2018 Guidelines for the Early Management of Patients With Acute Ischemic Stroke: A Guideline for Healthcare Professionals From the American Heart Association/ American Stroke Association. Stroke 2018;49:e46-e110.

12. Buck BH, Liebeskind DS, Saver JL, et al. Early neutrophilia is associated with volume of ischemic tissue in acute stroke. Stroke 2008;39:355-60.

13. McColl BW, Allan SM, Rothwell NJ. Systemic infection, inflammation and acute ischemic stroke. Neuroscience 2009; 158:1049-61.

14. Jickling GC, Liu D, Ander BP, et al. Targeting neutrophils in ischemic stroke: translational insights from experimental studies. J Cereb Blood Flow Metab 2015;35:888-901.

15. Tokgoz S, Kayrak M, Akpinar Z, et al. Neutrophil lymphocyte ratio as a predictor of stroke. J Stroke Cerebrovasc Dis 2013;22:1169-74.

16. Zhu B, Pan Y, Jing J, et al. Neutrophil counts, neutrophil ratio, and new stroke in minor ischemic stroke or TIA. Neurology 2018;90:e1870-8.

17. Song SY, Zhao XX, Rajah G, et al. Clinical Significance of Baseline Neutrophil-to-Lymphocyte Ratio in Patients With Ischemic Stroke or Hemorrhagic Stroke: An Updated Meta-Analysis. Front Neurol 2019;10:1032.

18. Hammoud K, Lanfranchi M, Li SX, et al. What is the diagnostic value of head MRI after negative head CT in ED patients presenting with symptoms atypical of stroke? Emerg Radiol 2016;23:339-44.

19. Plas GJ, Booij HA, Brouwers PJ, et al. Nonfocal Symptoms in Patients with Transient Ischemic Attack or Ischemic Stroke: Occurrence, Clinical Determinants, and Association with Cardiac History. Cerebrovasc Dis 2016;42:439-45.

20. Ishihara T, Sato S, Uehara T, et al. Significance of Nonfocal Symptoms in Patients With Transient Ischemic Attack. Stroke 2018;49:1893-8.

21. DeLong ER, DeLong DM, Clarke-Pearson DL. Comparing the areas under two or more correlated receiver operating characteristic curves: a nonparametric approach. Biometrics 1988;44:837-45.

22. Saber Tehrani AS, Coughlan D, Hsieh YH, et al. Rising annual costs of dizziness presentations to U.S. emergency departments. Acad Emerg Med 2013;20:689-96.

23. Newman-Toker DE, Hsieh YH, Camargo CA Jr, et al. Spectrum of dizziness visits to US emergency departments: cross-sectional analysis from a nationally representative sample. Mayo Clin Proc 2008;83:765-75.

24. Lee SH, Kim JS. Acute Diagnosis and Management of Stroke Presenting Dizziness or Vertigo. Neurol Clin 2015;33:687-98, xi.

25. Choi JH, Park MG, Choi SY, et al. Acute Transient Vestibular Syndrome: Prevalence of Stroke and Efficacy of Bedside Evaluation. Stroke 2017;48:556-62.

26. Paul NL, Simoni M, Rothwell PM. Transient isolated brainstem symptoms preceding posterior circulation stroke: a population-based study. Lancet Neurol 2013;12:65-71.

27. Pollak L, Shlomo N, Korn Lubetzki I. Headache in stroke according to National Acute Stroke Israeli Survey. Acta Neurol Scand 2017;135:469-75.

28. Madsen TE, Khoury J, Cadena R, et al. Potentially Missed Diagnosis of Ischemic Stroke in the Emergency Department in the Greater Cincinnati/ Northern Kentucky Stroke Study. Acad Emerg Med 2016;23:1128-35.

29. Coutts Shelagh B, Hill Michael D, Moreau F, et al. Abstract 99: Diagnosis of Uncertain Origin Benign Transient Events (DOUBT). Stroke 2018;49:A99.

30. Compter A, Kappelle LJ, Algra A, et al. Nonfocal symptoms are more frequent in patients with vertebral artery than carotid artery stenosis. Cerebrovasc Dis 2013;35:378-84.

31. Kim SH, Kim HJ, Kim JS. Isolated vestibular syndromes due to brainstem and cerebellar lesions. J Neurol 2017;264:63-9.

32. Li JW, Xu YY, Chen YJ, et al. Early elevated neutrophilto-lymphocyte ratio associated with remote diffusionweighted imaging lesions in acute intracerebral hemorrhage. CNS Neurosci Ther 2020;26:430-7.

33. Jayaraj RL, Azimullah S, Beiram R, et al. Neuroinflammation: friend and foe for ischemic stroke. J Neuroinflammation 2019;16:142.

34. Bhat T, Teli S, Rijal J, et al. Neutrophil to lymphocyte ratio and cardiovascular diseases: a review. Expert Rev Cardiovasc Ther 2013;11:55-9.

35. Boehme AK, Esenwa C, Elkind MS. Stroke Risk Factors, Genetics, and Prevention. Circ Res 2017;120:472-95. 
Page 10 of 10

36. Chase M, Goldstein JN, Selim MH, et al. A prospective pilot study of predictors of acute stroke in emergency department patients with dizziness. Mayo Clin Proc 2014;89:173-80.

Cite this article as: Wang ML, Yu MM, Li WB, Li YH. Application of neutrophil to lymphocyte ratio to identify CTnegative cerebral infarction with nonfocal symptoms. Ann Transl Med 2020;8(21):1359. doi: 10.21037/atm-20-1640

\section{Wang et al. NLR in cerebral infarction with nonfocal symptoms}

37. O'Donnell MJ, Xavier D, Liu L, et al. Risk factors for ischaemic and intracerebral haemorrhagic stroke in 22 countries (the INTERSTROKE study): a case-control study. Lancet 2010;376:112-23. 